\title{
Silica Nano-Networks as Stretches on Segmented SU8 Rods for Sub-Wavelength Photonics
}

\author{
Francois Doré $^{1}$, Bruno Bêche ${ }^{1}$, Nolwenn Huby ${ }^{1}$, F. Artzner ${ }^{1}$, Lionel Camberlein ${ }^{2}$, Etienne Gaviot ${ }^{2}$ \\ ${ }^{1}$ Institute of Physics of Rennes, UMR CNRS 6251, University of Rennes 1, Rennes, France \\ ${ }^{2}$ Laboratory of Acoustics, UMR CNRS 6613, University of Maine, Le Mans, France \\ E-mail: bruno.beche@univ-rennes1.fr, francois.dore@univ-rennes1.fr \\ Received January 20, 2011; revised March 4, 2011; accepted March 8, 2011
}

\begin{abstract}
We report an original approach based on a fluidic mechanism involving silica nano-particules that allowed us to design a elaborate set of segmented-optical structures such as arranged clusters of pillars and cross-tapered-waveguides. We show that the association of such complex segmented pre-formed structures can be specifically shaped, by way of coupling nano-fluidics and drying mechanisms. The formation of specific silica nano-patterns or organized networks of silica nano-ridges may be predictable and in perfect agreement with the theory of minimal surfaces dealt with physics of fluids. The interest of such nano-photonic coupling mechanisms has been clearly highlighted thanks to their abilities to build original nano-silica-networks and the specific development of new filters based on resonant tunnelling effects between multi-nano-ridges.
\end{abstract}

Keywords: Integrated Photonics, Nano-Connections, Silica Nano-Rib Waveguides, Nano-Optical Coupling, Nano-Network, Fluidic Mechanisms

\section{Introduction}

The main purpose of nano-optical-connections is to convey optical signals with a quite higher wavelength than the dimensions of a given optical waveguide arranged several wavelengths in the distance. Due to their noticeably high shape ratio (length/lateral-dimension), nano-waveguides structures as nanowires [1,2], nanotubes [3], nanoridges [4] and so on, present promising properties for optical components, especially light routing, nano-connections and networks. Thus, sub-wavelength waveguides are of great interest for investigation on sub-micronic propagation and optical nano-coupling mechanism between specific structures [5]. Regarding silica materials, various techniques based on new productions in materials science together with original processes proved the adequacy to develop hybrid integrated photonics and to obtain a noticeable nano-sized confinement marked with a spatial resolution around twenty times smaller than the proceeding wavelength [1-4]. Then, the ability to prepare silica nano-connections may open new opportunities for implementing low-dimensional silica materials. In a previous work [4], we have presented a fluidic mechanism coupled with silica nanoparticules so as to design single nano-ridge waveguides with two classical optical coupling configurations between organic straight-rib waveguides. In this paper, we highlight the great interest to process with such kind of nano-fluidic and dynamic dry devices. Indeed, being widened they come up as suitable to develop various and complex nano-connections with multi-sub- lambda branches and nano-networks between adequate segmented elements (tapers, pillars, rods). One of the key points is clearly that the pattern of such nano-networks as stretches on the abovementioned segmented elements is directly predictable and in perfect agreement with the theory of minimal surfaces dealt with classical physics of fluids [6]. Moreover, in such a complex nano-network, we have characterized the expected nano-optical coupling with a sub-wavelength routing propagation regime directly on the integrated chip.

\section{Silica Sub-Wavelength Connections and Networks Based on a Fluidic Approach Based on a Minimal Surface at Nanoscale}

Considering our processes and materials, relevant devices can be developed: They basically rely on a guided- 
wave proceeding on a (100) silicon substrate coated with a specific $\mathrm{SiO}_{2}$ layer first obtained by thermal oxidation of the silicon wafer, yielding $1 \mu \mathrm{m}$ in thickness with an index value $\mathrm{n}_{\mathrm{SiO} 2}$ close to 1.45 at visible and IR wavelengths. The organic SU8 film (whose higher refractive index 1.56 at such wavelengths is most suited for a guiding layer ranging around [1-10] $\mu \mathrm{m}$ in thickness), is deposited by spin coating and cured to remove the solvent according to convenient steps of temperature [7]. By way of UV-lithography, $40 \mathrm{~s}$ with UV light source (200 $\mathrm{mJ} / \mathrm{cm}^{2}$ ) and a similar baking modus to cross-link the polymer, adequate taper-waveguides and rod-pillars patterns defined on a mask are copied on the SU8 guiding layer. Then, a generic development process with the specific SU8 developer (MicroChem ${ }^{\circledR}$ ) allows us to obtain versatile structures prior to operate a mandatory postbaking $\left(200^{\circ} \mathrm{C}-2 \mathrm{~h}\right)$ so as to stabilize the SU8 global patterns several micrometers in thickness.

A specific mixture made of a sodium-dodecyl-sulfate surfactant (SDS) plus water is prepared with a concentration in silica beads (from Ludox ${ }^{\circledR}$ ) ranging around $5 \%$. The global solution of SDS with silica beads is deposited by way of a $\mu$ l-syringe onto a thin cover glass plate (devoted to optical microscopy). Then, the whole structure is turned down and carefully deposited onto the SU8 tapers and rod-pillars waveguides making up the optical chip patterns. Then, the nano-silica fluid can be strained between the elements of organic-structures: To this end, a drying mechanism is operated several minutes so as to shape a complex nano-network of silica nano-ridges, typically ranging around [50-100] nm in width, whose configuration is totally defined by the physics of fluids.

Figures 1(a) and 1(b) represent optical microscopy images respectively obtained by differential interference contrast (DIC) and scanning electron treatments of the nano-silica-connections after being stretched by drying fluid processes between the segmented SU8-tapers and -pillars. It may be noted as a key point, that with such a nanoscale scope, the formation of the specific silica nano-patterns or organized networks of silica nano- ridges is in perfect agreement with the theory of minimal surfaces provided with the mechanics of fluids. So, the nano-shape between the fourth rod pillar defined by two points and the couple of the three nano-ridges that emerge directly from both points at an angle of $120^{\circ}$ (Figure 1(b)) may be observed as a typical example: It is a specific shape accounting for a minimal surface [6] as regards a straight nano-ridge, if we consider the radii of the local curvature of the interface that tends to infinity with Laplace's equation. The interest of such processes and nano-fluidic mechanisms is clearly highlighted. Thanks to their ability to shape original nano- silica-networks, with a significant building potential in the third direction between more complex 3D-organic-

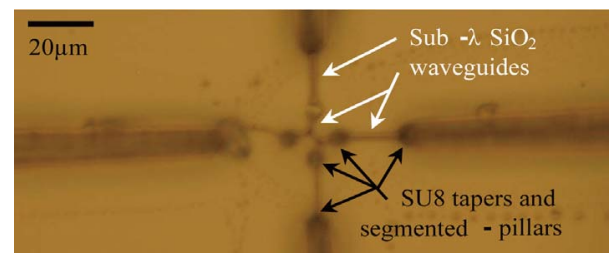

(a)

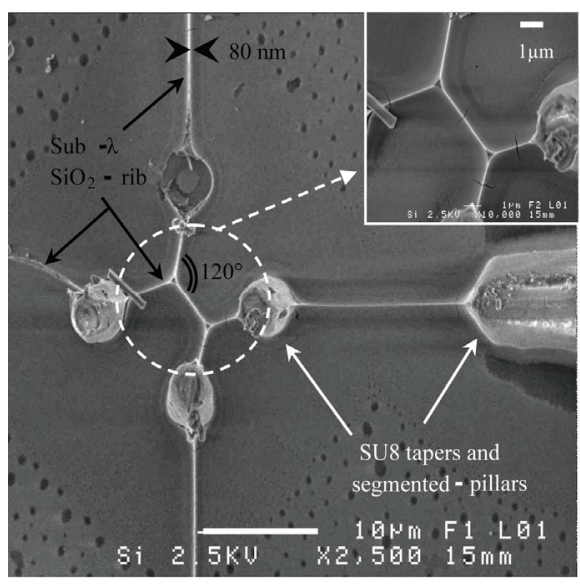

(b)

Figure 1. Optical microscopy images obtained by differential interference contrast (DIC) and scanning electron treatment of the nano-silica-connections stretched by drying fluid processes between segmented SU8-waveguides (tapers and pillars). The nano-pattern surrounded in white dashed line between the four SU8 pillars corresponds to the minimal surface shape obtained by fluidic mechanisms; as a result all the angles between straight nanoridge-waveguides that shape the nano-network are actually equal to $120^{\circ}$ : (a) general layout of the photonic structures; (b) silica nanophotonic network.

preforms obtained by advanced selective lithographic processes. Indeed 3D-structures may come up as far different from simple planar nano-networks.

\section{Nano-optical Coupling Imaging and Sub- wave-length Propagation}

Relevant photonic characterizations have been achieved by way of micro-injection processing with a specific optical bench so as to assess the performance of the obtained nano-network design related to the nanofluidic and minimal surface theory. Such a micro-optical injection bench consists of a laser source operating at $670 \mathrm{~nm}$ and micromanipulators associated with objectives so as to drive an upstream monomode optical field in the segmented SU8 taper operating as an integrated source connected with the downstream nano-network ridges. Hence, the excitation of the optical mode of the first SU8 taper-waveguide structure with its relevant nano-optical 


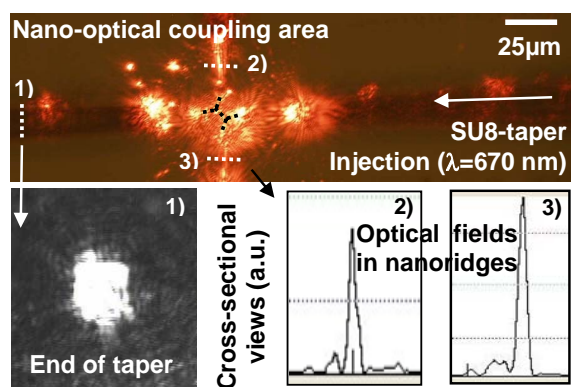

Figure 2. Photograph of a nano-optical coupling observed between the pre-formed SU8 tapers-pillars highlighting the sub-wavelength propagation regime located inside the silica nano-ridges for injection laser wavelengths $670 \mathrm{~nm}$. The image and graphs in the lower part depict the cross-sectional view and light intensity profiles of the optical field detected by a camera CCD respectively at the end of the SU8 waveguide opposite to the taper-injection, and with both perpendicular silica nano-ridges.

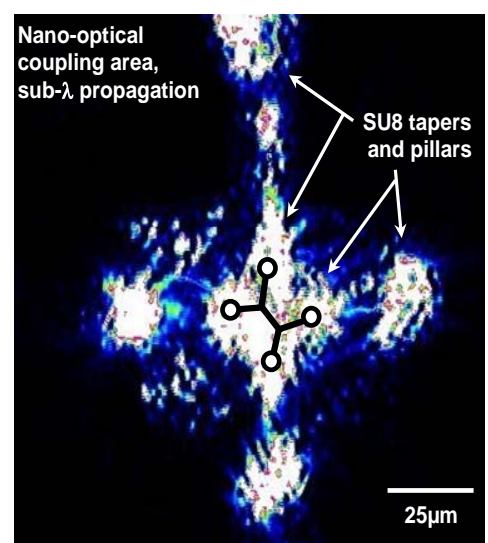

Figure 3. Upper-view and (zoom) of the nano-optical coupling located on the nano-patterns and sub-wavelength propagation area; the minimal surface or silica-nano-connections are shown with the black solid lines between the pillars.

coupling is verified, as well as the sub-wavelength propagation through the silica nanoridge-network (Figure 2).

A microscope and micro-beam profiler (MBP-100USB series from Newport ${ }^{\circledR}$ ) pitched on upper-view with its specific software allows us to characterize all the propagation mechanisms into the whole nano-ridges network. Cross-sectional views 2) and 3) in Figure 2 stand for the measurements and profile of the optical field into two given silica nano-rigdes.

The extremity of the bench is fitted with a (Pulnix$\mathrm{PE}$ )-camera, together with a video system so as to visualize and confirm the output single mode optical signal at the terminal of the SU8 taper (Figure 2, cross-sectional view 1) accounting for the sub-wavelength optical coupling and the propagation within the nano-network. Shown in Figure 3 is the nano-optical coupling area with sub-wavelength propagation through the minimal surface silica network depicted in Figures 1(a) and 1(b). Then, such effective propagation and nano-coupling mechanisms have been validated into complex silica nano-net works fabricated with nanofluidic processes.

\section{Conclusion}

We have validated the experimental mainstays regarding a hybrid-organic/inorganic-materials approach, based on combining a system of specific fluids loaded with nano-particles together with adequate organic integrated optical processes: Then we have demonstrated the ability to shape complex silica 2D-nano- networks as stretches arranged on segmented pre- formed SU8 rods-pillars and tapers devoted to sub- wavelength photonics. The formation of such specific silica patterns at nanoscale, or organized networks of silica nano-ridges, may be totally predictable and in total agreement with the so-called theory of minimal surfaces addressed by physics of fluids.

Considering micro-optical injection, specific coupling mechanisms allowed us to observe a sub-wavelength propagation regime into operative silica nano-connections, a network being arranged upon a set of preformed-patterned rods/tapers. Such reproducible technologies come up as a low-cost and interesting solution to shape versatile and complex 3D-nano-netwoks for future nano-optical routing schemes by using advanced and selective technical-lithographies on previously preformed organics.

\section{Acknowledgements}

The authors would like to express their gratitude Rennes Métropole, Région Bretagne and ANR-08-JCJC0136-01 programs for their financial support. The authors gratefully acknowledge Pr. I. Cantat (IPR UMR CNRS 6251, France) for their kind discussions on fluidic processes.

\section{References}

[1] L. Tong, R. R. Gattass, J. B. Ashcom, S. He, J. Lou, M. Shen, I. Maxwell and E. Mazur, "Subwavelength-Diameter Silica Wires for Low-Loss Optical Wave Guiding”, Nature, Vol. 426, No. 4968, 2003, pp. 816-819. doi:10.1038/nature02193

[2] L. Tong, J. Lou, R. R. Gattass, S. He, X. Chen, L. Liu and E. Mazur, "Assembly of Silica Nanowires on Silica Aerogels for Microphotonic Devices,” Nano Letters, Vol. 4, No. 2, 2005, pp. 259-262. doi:10.1021/nl0481977

[3] D. Duval, C. Tarabout, F. Artzner, E. Gaviot, A. Renault and B. Bêche, "Development of New Practical Approach 
to Integrated Photonics based on Biomimetic Molecular Self-Assembled Nanotubes,” Electronics Letters, Vol. 44, No. 19, 2008, pp. 1134-1135. doi:10.1049/el:20081672

[4] B. Bêche, A. Jimenez, L. Courbin, L. Camberlein, F. Artzner and E. Gaviot, "Functional Silica Nano-Connections Based on a Fluidic Approach for Integrated Photonics,” Electronics Letters, Vol. 46, No. 5, 2010, pp. 356-357. doi:10.1049/el.2010.2569

[5] L. Dobrzynski, B. Djafari-Rouhani, A. Akjouj, J. O. Vasseur and J. Zemmouri, "Photonic Tunnelling between Two Wires,” Progress in Surface Science, Vol. 67, No. 1-8,
2001, pp. 347-354. doi:10.1016/S0079-6816(01)00035-1

[6] P. G. De Gennes, F. Brochard-Wyart and D. Quéré, “Gouttes, Bulles, Perles et Ondes,” Editions Belin, 2002.

[7] B. Bêche, N. Pelletier, E. Gaviot and J. Zyss, "SingleMode TE00-TM00 Optical Waveguides on SU8 Polymer," Optics Communications, Vol. 230, No. 1-3, 2004, pp. 91-94. doi:10.1016/j.optcom.2003.11.016 